\title{
Circular RNA circYPEL2: A Novel Biomarker in Cervical Cancer
}

\author{
Xinyang Zhang ${ }^{1,+}{ }^{,}$, Siqi Yang ${ }^{1,+}$, Wenbo Chen ${ }^{1}\left(\mathbb{D}\right.$, Xin Dong ${ }^{1}$, Rongyu Zhang ${ }^{2}$, Haidong Ye $^{2}$, Xiangfei Mei ${ }^{1}$, \\ Huan Liu ${ }^{3, *}$, Yu Fang ${ }^{2, *}$ and Chunjiang $\mathrm{He}^{2}$ (I) \\ 1 School of Basic Medical Sciences, Wuhan University, Wuhan 430071, China; \\ zhangxinyang@whu.edu.cn (X.Z.); smyangsiqi@163.com (S.Y.); cwb528@whu.edu.cn (W.C.); \\ dongxin@whu.edu.cn (X.D.); 2020203010023@whu.edu.cn (X.M.) \\ 2 College of Biomedicine and Health, Huazhong Agricultural University, Wuhan 430070, China; \\ zry@webmail.hzau.edu.cn (R.Z.); 14779862806@163.com (H.Y.); che@whu.edu.cn (C.H.) \\ 3 University of Science and Technology of China, Hefei 230026, China \\ * Correspondence: liuhuan520@ustc.edu.cn (H.L.); fangyu_jy@whu.edu.cn (Y.F.) \\ + These authors contributed equally to this work.
}

check for updates

Citation: Zhang, X.; Yang, S.; Chen, W.; Dong, X.; Zhang, R.; Ye, H.; Mei, X.; Liu, H.; Fang, Y.; He, C. Circular RNA circYPEL2: A Novel Biomarker in Cervical Cancer. Genes 2022, 13, 38. https: / / doi.org/10.3390/ genes13010038

Academic Editors: Xiang Zhou, Xiaoli Zhang and Björn Voß

Received: 8 November 2021 Accepted: 17 December 2021 Published: 23 December 2021

Publisher's Note: MDPI stays neutral with regard to jurisdictional claims in published maps and institutional affiliations.

Copyright: (C) 2021 by the authors. Licensee MDPI, Basel, Switzerland. This article is an open access article distributed under the terms and conditions of the Creative Commons Attribution (CC BY) license (https:// creativecommons.org/licenses/by/ $4.0 /$ )

\begin{abstract}
Cervical cancer (CC) is one of the most threatening diseases in women. Circular RNAs (circRNAs) have been reported to be cancer hallmarks, but typical circRNAs in CC were rarely indicated. Through high-throughput sequencing in CC and normal cervix tissues, circYPEL2 (hsa_circ_0005600) was proposed as a candidate circRNA. CircYPEL2 exhibited significantly high expression in CC tissue and strong stability in CC cell lines. Furthermore, knockdown and overexpression of circYPEL2 indicated the potential involvement in CC proliferation, migration and invasion. Finally, the downstream regulatory genes of circYPEL2 were investigated by knockdown experiment in CC cell lines with high-throughput sequencing. In summary, our work identified circYPEL2 as a potential biomarker for clinical research of cervical cancer.
\end{abstract}

Keywords: cervical cancer; circRNA; biomarker

\section{Introduction}

Cervical cancer (CC) is the fourth most common cancer in women, causing death in up to $55 \%$ of patients. Human papillomavirus (HPV) infection is the major factor underlying CC [1]. More than 100 types of HPV have been identified as high-risk viruses based on their oncogenic characteristics, among which HPV 16 and HPV 18 are the most critical carcinogenic subtypes [2]. With the widespread application of vaccines against HPV and early cancer screening, an increasing number of patients can be treated at the early stages of the disease [3]. Nevertheless, the current method for CC screening is dependent on microscopic examination of cervical cells and HPV testing, which includes several complicated steps and is costly [4]. Identifying novel biomarkers for CC screening is a candidate procedure to improve the accuracy of diagnosis.

As a new family of noncoding RNAs, circular RNAs (circRNAs) are formed by backsplicing of exons/introns. Compared to linear transcripts, circRNAs do not contain a $5^{\prime}$ terminal cap structure or $3^{\prime}$-terminal poly A, rendering them more resistant to exonucleases and leading to relatively high stability in tissue and cells [5-8]. CircRNAs have been widely reported exerting functions as competing endogenous RNAs (ceRNAs). In addition, circRNAs can interact with RNA-binding proteins [9] and host DNA [10], and may translate functional proteins [11]. CircRNAs have been indicated as hallmarks in many cancers. For example, circFOXO3 can activate the expression of host gene FOXO3 by binding with p53 and MDM2, resulting in the increased apoptosis of breast cancer cells [12]. CircMTO1 was significantly downregulated in HCC and correlated with the survival of HCC patients [13]. CircTLK1 exacerbates renal cancer by activating CBX4 via competitive interaction with miR-136-5p [14]. CircSLC8A1 has been defined as a potential biomarker for the diagnosis of bladder cancer [15]. Though circRNAs have been revealed playing important roles in tumorigenesis, the function and regulation mechanism of circRNAs in CC are less known. 
In this study, we performed expression profiling of circRNA in clinical CC and normal control samples using high-throughput sequencing and identified a CC-associated circRNA circYPEL2, which promoted the proliferation, invasion and migration of CC cells. Knockdown of circYPEL2 regulated downstream genes. Our work indicated that circYPEL2 may act as a potential biomarker in the development of CC.

\section{Materials and Methods}

\subsection{Sample Treatment and Cell Culture}

$\mathrm{CC}$ and normal control tissues were collected from CC patients and healthy volunteers from Wuhan University Zhongnan Hospital (Wuhan, China). Total RNA was extracted for whole transcriptome sequencing. RNA sequencing was performed using an Illumina HiSeq 2000, and all raw data were deposited in the Gene Expression Omnibus (GEO) repository (https: / / www.ncbi.nlm.nih.gov / geo/query/acc.cgi, accession number GSE173112). The CC cell lines HeLa and SiHa, and human normal cell line 293T were maintained in DMEM (HyClone, Los Angeles, CA, USA), supplemented with 10\% fetal bovine serum (Gibco, Carlsbad, CA, USA) and 1\% penicillin-streptomycin (Gibco, Carlsbad, CA, USA) and cultured at $37{ }^{\circ} \mathrm{C}$ in a humidified $5 \% \mathrm{CO}_{2}$ incubator.

\subsection{Processing of Sequencing Data}

Low-quality reads and sequencing adapters were removed by Trim-Galore to obtain clean data. Then, the circular transcripts were identified and the circular/linear ratios (CLR) were calculated by CLEAR [16]. The linear transcripts were mapped to the hg19 genome by STAR [17] and counts were quantified using featureCounts [18]. TPM of linear transcripts were calculated based on mapped read counts $[19,20]$. Differential expression analysis of circRNA and mRNA was performed using the DESeq2 package [21], genes with $\mid$ Log2Fold Change $\mid>1$ and adjusted $p$-value $<0.05$ were considered as differentially expressed. Pheatmap package was used for clustering analysis. GO enrichment analysis was performed using the clusterProfiler [22]. The interactions of the downstream genes of circRNA were obtained from STRING (https:/ / cn.string-db.org/, accessed on 1 September 2021).

\subsection{Total RNA and Genomic DNA Isolation}

Total RNA was extracted using TRIzol reagent (Invitrogen, Carlsbad, CA, USA) according to the manufacturer's instructions. The gDNA was extracted using a TIANamp Genomic DNA Kit (TIANGEN, Beijing, China).

\subsection{RT-PCR and $q P C R$}

Total RNA was reverse-transcribed using a Hifair ${ }^{\circledR}$ II 1st Strand cDNA Synthesis Kit (gDNA digester plus) (Yeasen, Shanghai, China) according to the manufacturer's instructions. For PCR, $2 \times$ Hieff $^{\mathrm{TM}}$ PCR Master Mix (With Dye) (Yeasen, Shanghai, China) was used. The cDNA and gDNA PCR products were evaluated using $2 \%$ agarose gel electrophoresis. The qPCR was conducted using $2 \times$ SYBR Green qPCR Master Mix (Bimake, Houston, TX, USA). GAPDH, $\beta$-actin and U1 were used as controls, and relative expression levels were calculated using the $2^{-\Delta \Delta \mathrm{Ct}}$ formula. All primer sequences are listed in Supplementary Table S1.

\subsection{RNase $R$ and Actinomycin D Assay}

The $2 \mu \mathrm{g}$ RNA was incubated at $37^{\circ} \mathrm{C}$ for $15 \mathrm{~min}$ with or without $3 \mathrm{U} / \mu \mathrm{g}$ RNase R (Lucigen, Ogden, UT, USA), and inactivated at $70^{\circ} \mathrm{C}$ for $10 \mathrm{~min}$, then analyzed by RT-PCR. $\mathrm{HeLa}$ and SiHa cells were incubated in DMEM with or without $2 \mu \mathrm{g} / \mathrm{mL}$ actinomycin D (MCE, Jersey, NJ, USA) for $24 \mathrm{~h}$ before RNA extraction [23,24]. The expression of circYPEL2 and YPEL2 mRNA was analyzed using RT-PCR. 


\subsection{Fractionation of Nuclear and Cytoplasma}

Approximately $10^{6}-10^{7}$ cells were collected, washed with ice-cold PBS twice and resuspended in $300 \mu \mathrm{L}$ lysis buffer (1\% NP-40, 0.5\% sodium deoxycholate, $5 \mathrm{mM}$ EDTA, $1 \mathrm{mM}$ DTT, $1 \mathrm{mM}$ PMSF, $2 \mathrm{mM}$ VRC, 15\% glycerol, $1 \times$ proteinase inhibitor cocktail), incubated on ice for $5 \mathrm{~min}$ and centrifuged at $4000 \times \mathrm{g}$ for $1 \mathrm{~min}$ at $4{ }^{\circ} \mathrm{C}$, and the supernatant was saved for cytoplasmic fractionation. The precipitated nuclei were further washed once with lysis buffer, resuspended in $300 \mathrm{~mL}$ lysis buffer, sonicated and centrifuged at $13,000 \mathrm{rpm}$ for $10 \mathrm{~min}$ at $4{ }^{\circ} \mathrm{C}$. The supernatant was saved as the nuclear extract.

\subsection{Plasmid Construction and Cell Transfection}

For circYPEL2 overexpression, the sequence for exon 2 of YPEL2 was amplified using PrimerSTAR Max DNA Polymerase Mix (Takara, Dalian, China) and then inserted into pLCDH-ciR (Geenseed, Guangzhou, China), which was reconstructed by inserting a front circular frame and back circular frame to promote RNA circularization. Cells were transfected using Zlipo2000 (Zomanbio, Beijing, China) and PepMute (SignaGen, Baltimore, MD, USA). All primers and oligonucleotide sequences are listed in Supplementary Table S1.

\subsection{Colony Formation Assay}

HeLa and SiHa cells transfected with the plasmids or siRNA were cultured in 6-well plates at a density of 300-500 cells per well. Plates were incubated at $37{ }^{\circ} \mathrm{C}$ in $5 \% \mathrm{CO}_{2}$ for 10-14 days, and colonies with more than 50 cells were scored. Cell colonies were immobilized with $4 \%$ paraformaldehyde (Biosharp, Hefei, China), stained with $0.5 \%$ crystal violet solution (Solarbio, Beijing, China), and then visualized under a microscope.

\subsection{CCK-8 Assay}

The proliferation of CC cells was determined using Cell Counting Kit-8 (CCK-8, Biosharp, Hefei, China) according to the manufacturer's instructions. In brief, HeLa and SiHa cells were seeded into 96-well plates at a density of 2000 cells / well after transfection. Then, $10 \mu \mathrm{L}$ CCK-8 solution was added to each well after 24,48 and $72 \mathrm{~h}$. The absorbance was measured at a wavelength of $450 \mathrm{~nm}$ by a Multiskan FC Microplate Photometer (Thermo Fisher, Waltham, MA, USA).

\subsection{Cell Migration and Invasion Assay}

Transwell assays were used to evaluate the invasion and migration capacities of CC cells in vitro. For cell migration, HeLa and SiHa cells were harvested after transfection for $24 \mathrm{~h}$. A total of $2 \times 10^{4}$ cells were seeded in the upper chamber with $500 \mu \mathrm{L}$ serumfree medium, and medium containing 10\% FBS was added into the lower chamber as a chemoattractant. For the cell invasion assay, $100 \mu \mathrm{L}$ Matrigel was added to the upper chamber. After incubation for $24 \mathrm{~h}$, cells on the upper surface of the membrane were removed by wiping with a Q-tip, and the invaded or migrated cells were immobilized with $4 \%$ paraformaldehyde (Biosharp, Hefei, China) and stained with $0.5 \%$ crystal violet solution (Solarbio, Beijing, China). Then, positive cells were quantified.

\subsection{Statistical Analysis}

For qPCR, colony formation assay, cell migration and invasion assay, all these experiments were independently conducted three times. Relative expression or cell number were presented as the mean \pm SD. Statistical analyses were performed using GraphPad Prism 7.0 (La Jolla, San Diego, CA, USA). The Student unpaired $t$-test was performed to identify the differences between two experimental groups. For CCK-8 assay, experiments were independently conducted three times and six technical repetitions were performed at each time point. OD450 values were presented as the mean \pm SD. Two-way ANOVA was performed to identify the effect of two factors on cell growth and multiple comparison adjustment was performed by Benjamin-Hochberg algorithm for the comparison at each point time. Statistical analyses were performed using GraphPad Prism 7.0 (La Jolla, San 
Diego, CA, USA) and R (www.r-project.org, accessed on 4 September 2021). As statistical significance, ${ }^{*} p<0.05,{ }^{* *} p<0.01,{ }^{* * *} p<0.001$ and ${ }^{* * * *} p<0.0001$ were considered.

\section{Results}

\subsection{Identification of CC-Associated circRNAs}

We performed RNA-seq for three CC tissues and three normal cervical samples and identified circRNAs. Results indicated a total of 93,509 circRNAs were detected in CC and normal samples. Overlap analysis revealed 59,093 circRNAs are only expressed in CC and 22,188 circRNAs are only expressed in normal samples (Figure 1A). In total circRNAs, $68,107(76 \%)$ circRNAs were derived from the exonic type, while $11,060(12 \%)$ and 10,520 $(12 \%)$ circRNAs were derived from intronic and intergenic regions, respectively (Figure 1B). In addition, we calculated the ratio of circular to linear transcripts (CLR) in tumor and normal tissues. Results showed that CLR in CC tissues was significantly lower than that in normal controls, which was identical to previous works $[8,25]$, revealing an average lower expression of circRNAs in cancers (Figure 1C). 

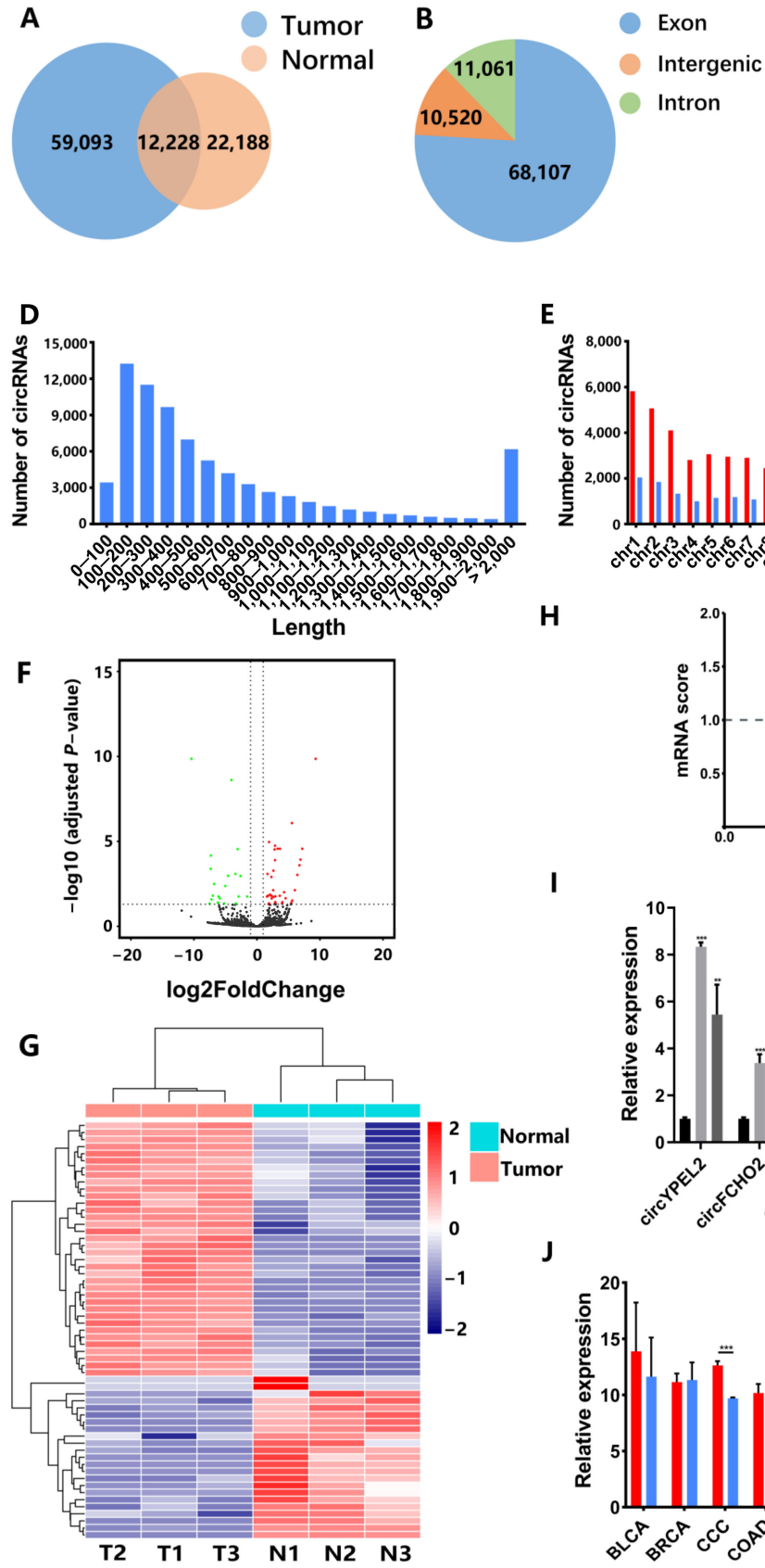

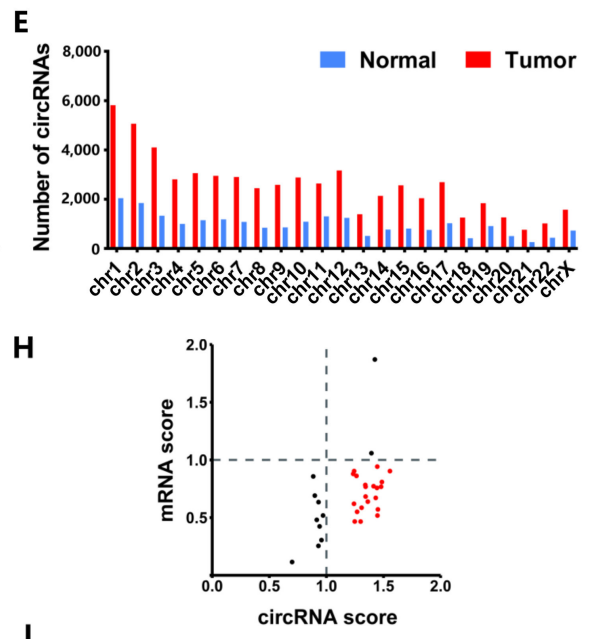

I
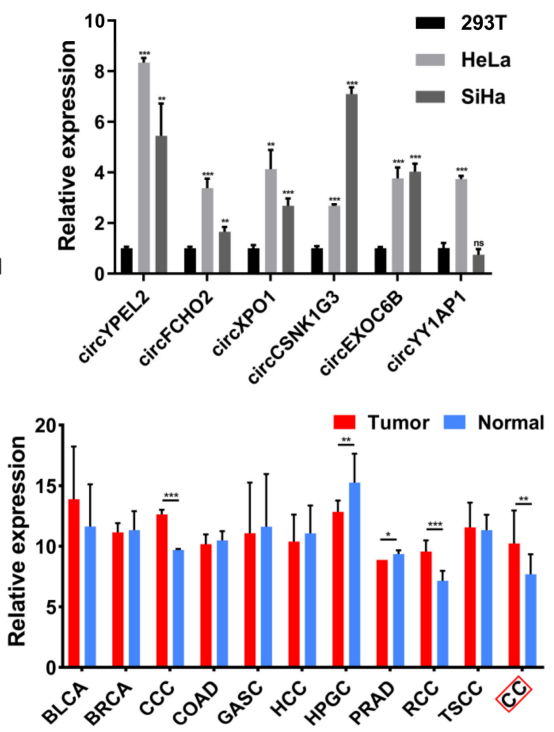

Figure 1. Identification of circRNA expression in CC. (A) Number of circRNAs detected in tumor (3 samples) and normal tissues (3 samples). (B) The gene location of circRNAs. (C) The ratios of circular and linear RNA expression in tumor (3 samples) and normal tissues (3 samples) $\left(p\right.$-value $\left.<2.2 \times 10^{-16}\right)$. (D) The length distribution of circRNAs. (E) The chromosome locations of circRNAs. (F) Volcano plot of differentially expressed circRNAs in CC. Red and green points represent significantly upregulated and downregulated circRNAs, respectively $(\mid \log 2$ FoldChange $\mid>1$, adjusted $p$-value $<0.05)$. (G) Clustering analysis of circRNAs with significantly different expression between tumor and normal samples. Normalized counts from DESeq2 were used for depicting the heatmap. (H) The anchor of circYPEL2. Points represent differentially expressed circRNAs ( $\mid \log 2$ FoldChange $\mid>1$, adjusted $p$-value $<0.05$, average expression $>25$ ). Red points represent circRNAs that were upregulated in tumors, while their host genes were downregulated. (I) RT-PCR of differentially expressed circRNA in HeLa and SiHa cell lines. (J) Expression of circYPEL2 in various cancers based on CSCD2 database. (Mean \pm SD of three experimental replicates are presented. * Represents $p<0.05,{ }^{* *}$ represents $p<0.01,{ }^{* * *}$ represents $p<0.001$ ). 
The length of circRNA transcripts were mostly from $100 \mathrm{bp}$ to $700 \mathrm{bp}$ (Figure 1D). In addition, the chromosomal distribution of circRNA transcripts exhibited that there were more circRNAs in CC than normal samples in all chromosomes (Figure 1E). Location analysis exhibited most of circRNAs were derived from encoded genes (Supplementary Figure S1A).

A total of 59 circRNAs were detected with significantly differential expression between $\mathrm{CC}$ and normal samples ( $\mid \log 2$ FoldChange $\mid>1$ and adjusted $p$-value $<0.05$ ). Among them, 36 circRNAs were upregulated and 23 circRNAs were downregulated in tumor tissues (Figure 1F,G and Supplementary Figure S1B). Notably, we observed 65.6\% highly expressed circRNAs derived from lowly expressed host genes in CC (Figure 1H, red points). To verify the RNA-seq results, qPCR for these circRNAs were conducted in HeLa and SiHa cells, with 293T cell line, which is a widely used normal control according to previous works [26-29]. Results showed the expression of circYPEL2 was highest in seven circRNAs, which were consistent to RNA-seq results (Fold-change $>6$, and adjusted $p$-value $=0.0090$, Student unpaired $t$-test) (Figure 1I and Supplementary Figure S1D).

Next, we detected the expression of circYPEL2 in CSCD2 database [30], which includes circRNA expression in most of cancer types, and observed circYPEL2 is differentially expressed in cervical cancer (CC), as well as several other malignancies, including clear cell carcinoma (CCC), hypopharyngeal cancer (HPGC), prostate cancer (PRAD) and renal cell carcinoma (RCC) (Figure 1J), suggesting that circYPEL2 may play an important role in the development of cancers.

\subsection{CircRNA Exerts Strong Stability in CC Cell Lines}

CircYPEL2 is derived from exon 2 (312 bp) of YPEL2. The back-spliced junction site of circYPEL2 was amplified using divergent primers and confirmed by Sanger sequencing (Figure 2A). PCR using convergent primers and divergent primers amplifying linear and circular RNA was conducted in cDNA and genomic DNA (gDNA), respectively in HeLa and SiHa cells, and circYPEL2 was only amplified by divergent primers in CDNA but not in gDNA (Figure 2B). In addition, circYPEL2 exhibited resistance while the linear transcript exhibited significant degradation after treatment by RNase R (Figure 2C). Next, HeLa and $\mathrm{SiHa}$ cells were pretreated with actinomycin D, a transcription inhibitor, for $24 \mathrm{~h}$, followed by qRT-PCR analysis. Comparing to the linear transcript, circYPEL2 exhibited strong resistance to actinomycin $\mathrm{D}$, indicating distinct biological stability (Figure 2D). In addition, fractionation experiment of nuclear and cytoplasmic revealed that circYPEL2 was prominently localized in the cytoplasm rather than the nucleus (Figure 2E). These results revealed the features of circYPEL2 in CC cells and suggested a potential stable regulator in tumorigenesis.

\subsection{Knockdown of circYPEL2 Attenuates the Proliferation, Migration and Invasion of CC}

To investigate the regulatory mechanism of circYPEL2 in CC, transfection of short interfering RNAs (si-NC, si-circYPEL2-1, si-circYPEL2-2) was performed (Figure 3A). SicircYPEL2-2 was selected for subsequent experiments due to its increased efficiency and specificity of interference (Figure 3B). The results of CCK-8 and colony formation assays indicated that the viability and proliferation of CC cells were inhibited by circYPEL2 knockdown (Figure 3C,D). Furthermore, transwell assays revealed significant abatement of cell migration and invasion upon circYPEL2 silencing (Figure 3E,F). In summary, knockdown of circYPEL2 attenuated the proliferation, migration and invasion of CC.

\subsection{Overexpression of circYPEL2 Promotes the Proliferation, Migration and Invasion of CC}

To further confirm the function of circYPEL2, we constructed a circYPEL2 overexpression plasmid using the pLCDH-ciR vector (Figure $4 \mathrm{~A}$ ), which contains a front and back circular frame. The qRT-PCR analysis was then performed to ensure overexpression efficiency (Figure 4B). Next, the viability and proliferation of CC cells were evaluated. Cell proliferation was activated by pLCDH-circYPEL2 transfection (Figure 4C,D), and cell migration and invasion ability were induced as measured by transwell assay. Exoge- 
nous supplementation with circYPEL2 obviously contributed to migration and invasion (Figure 4E,F). These results further indicated that circYPEL2 promoted the progression of CC.

A

chr17:57,430,575-57,430,887(GRCh37/hg19)
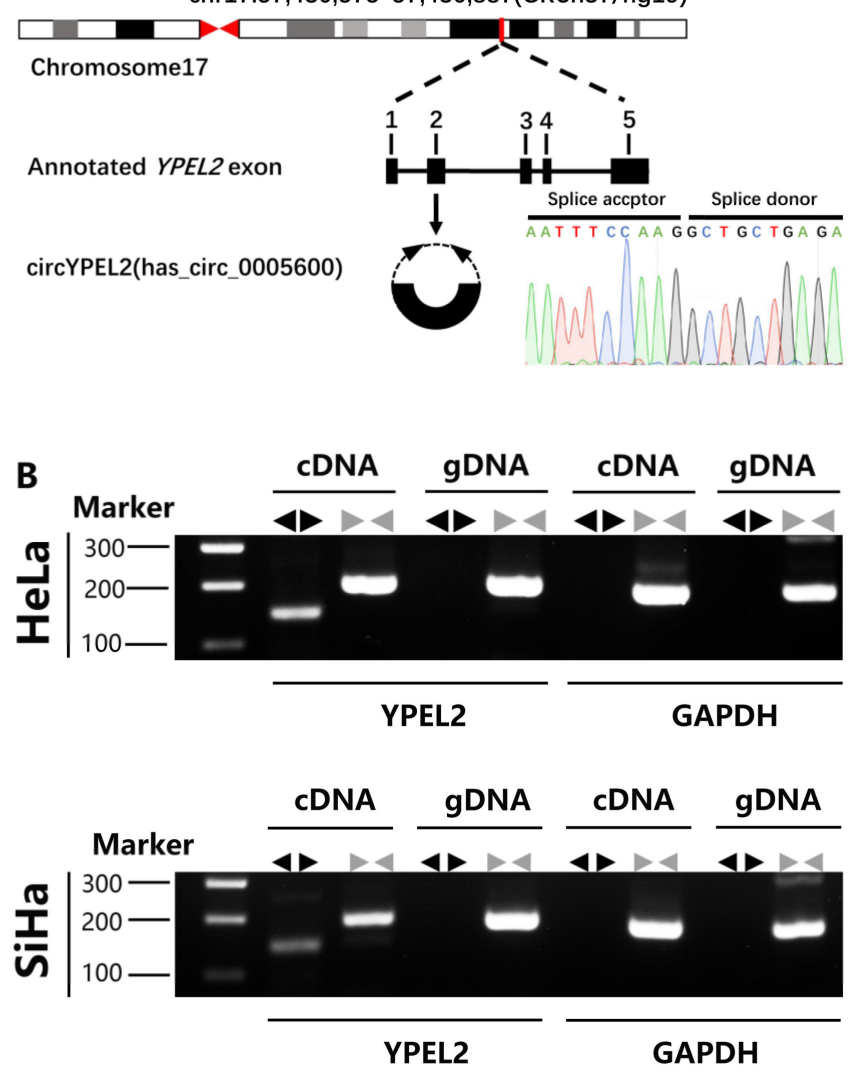

C
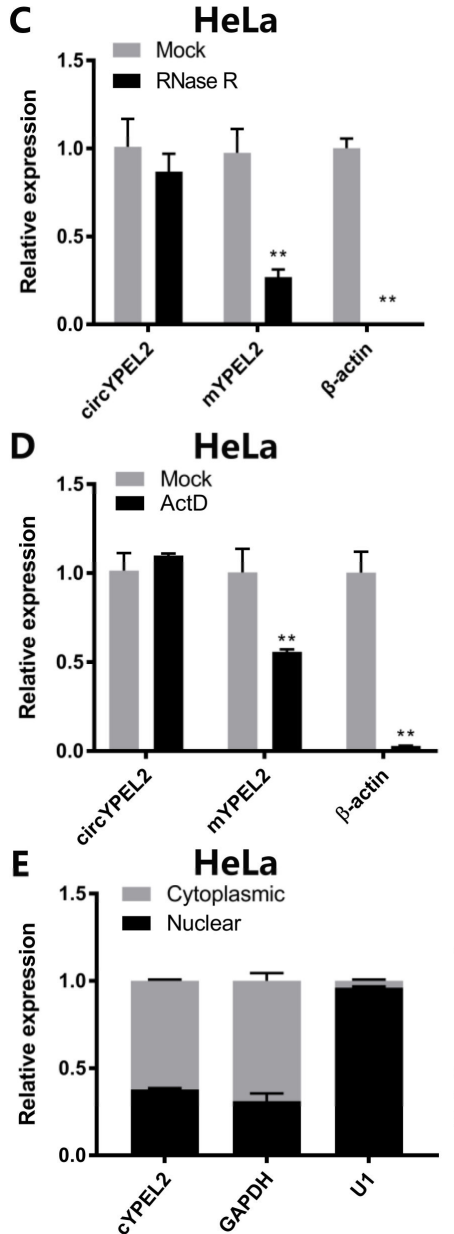

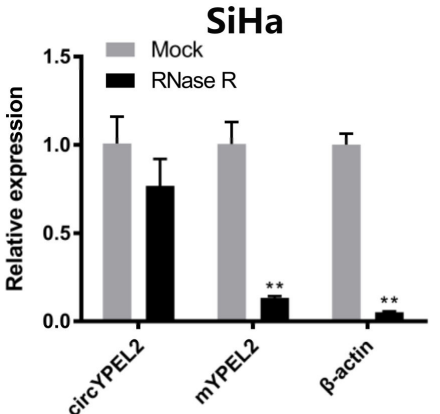

$\mathrm{SiHa}$
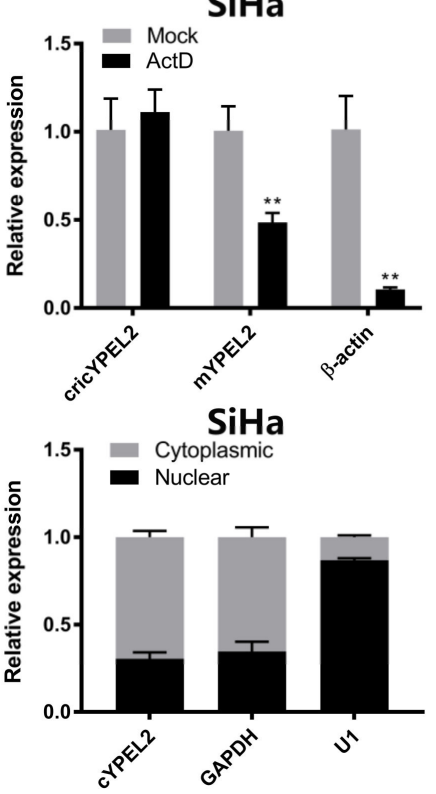

Figure 2. CircYPEL2 exhibits stability in CC cell lines. (A) Structures of the YPEL2 genome and transcript. CircYPEL2 is derived from exon 2. The back-splice junction of circYPEL2 was identified by Sanger sequencing. (B) RT-PCR assay with divergent or convergent primers indicated the expression of circYPEL2 in HeLa and SiHa cell lines. GAPDH was used as negative control. The cDNA: complementary DNA; gDNA: genomic DNA. (C) The qRT-PCR analysis of the expression of circYPEL2 and YPEL2 mRNA after treatment with RNase R in HeLa and SiHa cell lines. (D) The qRT-PCR analysis of the expression of circYPEL2 and YPEL2 mRNA in response to treatment with actinomycin D for $24 \mathrm{~h}$ in HeLa and SiHa cell lines. (E) Fractionation experiments of cytoplasmic and nuclear mRNA showed that circYPEL2 localized in the nucleus and cytoplasm. $\beta$-actin and U1 were applied as positive controls in the cytoplasm and nucleus, respectively. (Mean $\pm \mathrm{SD}$ of three experimental replicates are presented. ${ }^{* *}$ Represents $\left.p<0.01\right)$.

\subsection{CircYPEL2 May Regulate CC via Downstream Genes}

To explore the potential regulatory mechanism of circYPEL2 in CC, we performed RNA-seq in HeLa and SiHa cell lines with knockdown of circYPEL2. Differential expression analysis revealed that 22 genes were significantly regulated by circYPEL2 knockdown in two cells (Figure 5A). RT-PCR was used to validate the significantly differentially expressed genes (Figure 5B). Gene Ontology analysis indicated that most of the differentially expressed genes were enriched in leukocyte differentiation, blood circulation and cell differentiation, which are correlated with proliferation and migration (Figure 5C). In addition, we observed these genes are enriched in GSEA hallmark path- 
ways (https:/ / www.gsea-msigdb.org/gsea/index.jsp, accessed on 15 September 2021), such as epithelial-mesenchymal transition (EMT) and KRAS signaling, as well as oxidative phosphorylation, hypoxia and reactive oxygen pathways, which are associated with tumorigenesis and energy metabolism [31-33] (Supplementary Table S2). Furthermore, the interaction network between these downstream genes of circYPEL2 was predicted (Figure 5D).

A

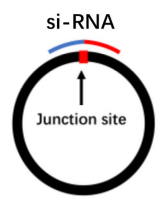

si-NC

si-circYPEL2-1 5'-GAACUAAUUUCCAAGGCUGTT-3' si-circYPEL2-2 5'-UUCCAAGGCUGCUGAGAACTT-3'

\section{C}

HeLa

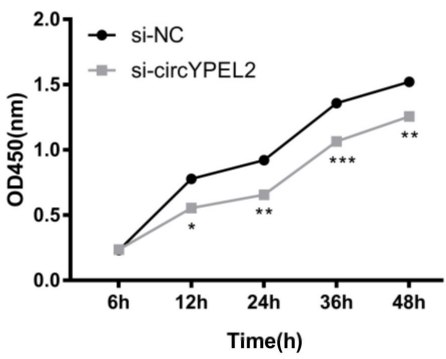

$\mathbf{E}$

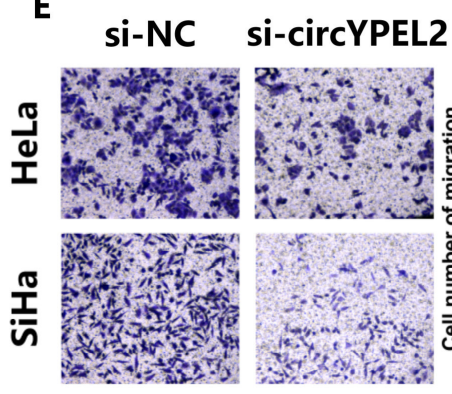

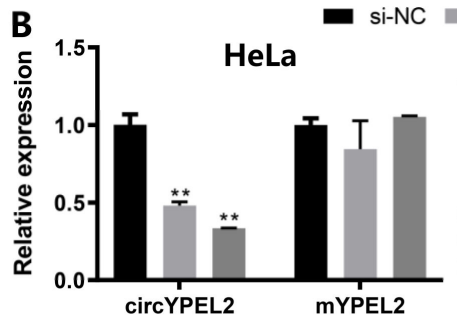

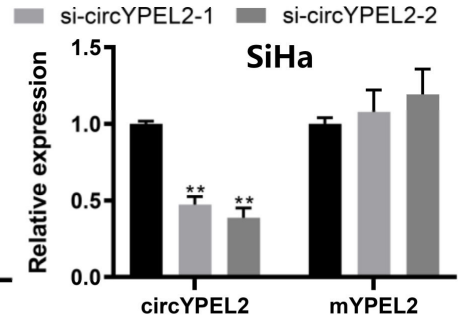

D Si-NC si-circYPEL2
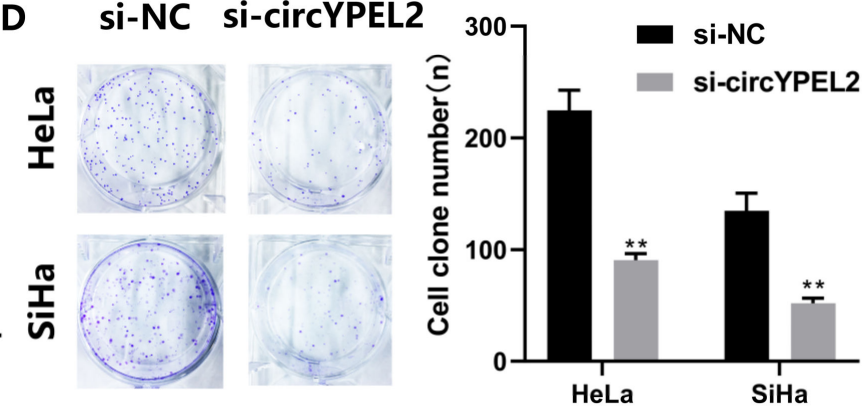

$\mathbf{F}$
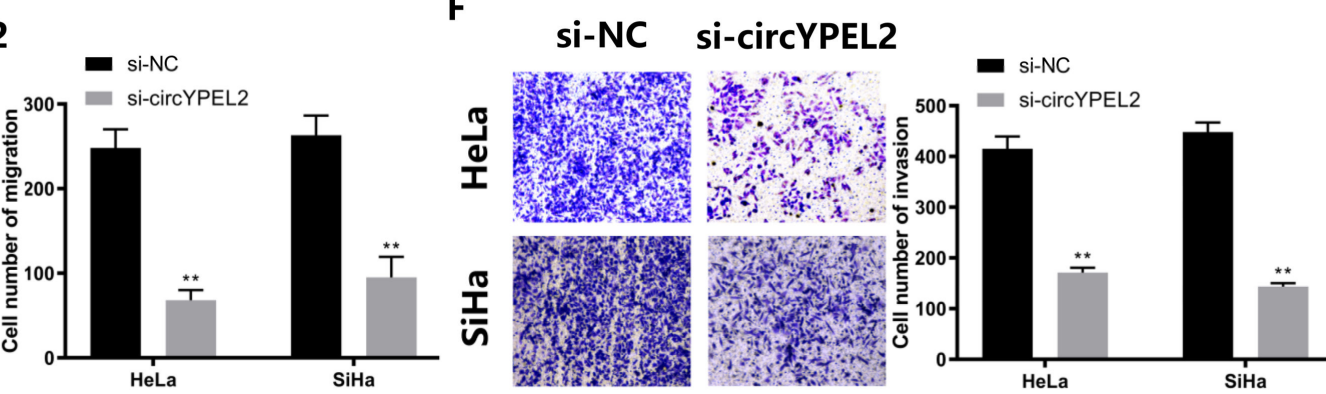

Figure 3. Knockdown of circYPEL2 suppresses cell proliferation, migration and invasion. (A) Schematic illustration and sequences of si-NC and si-circYPEL2. (B) Expression levels of circYPEL2 and YPEL2 mRNA in HeLa and SiHa cell lines transfected with si-circYPEL2. Si-NC was the negative control. (C) CCK-8 assays in HeLa and SiHa cell lines to detect cell viability at 0, 6, 12, 24, 36 and $48 \mathrm{~h}$. (D) Clone formation assays in HeLa and SiHa cell lines to detect cell proliferation. (E,F) Transwell assay for cell migration and invasion ability in HeLa and SiHa cells. (Mean $\pm \mathrm{SD}$ of three experimental replicates are presented. * Represents $p<0.05,{ }^{* *}$ represents $p<0.01,{ }^{* * *}$ represents $p<$ $0.001,{ }^{* * * *}$ represents $\left.p<0.0001\right)$. 
A

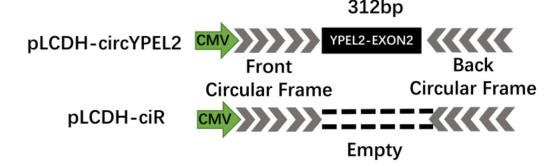

B

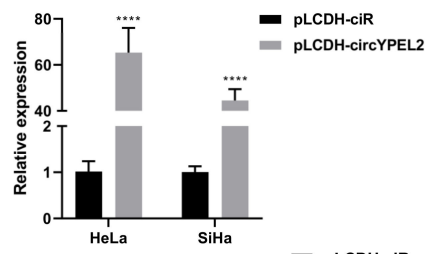

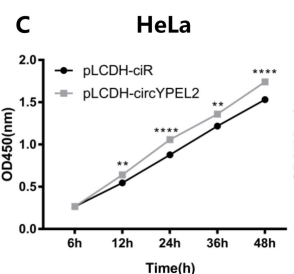

E pLCDH-ciR pLCDH-circYPEL2
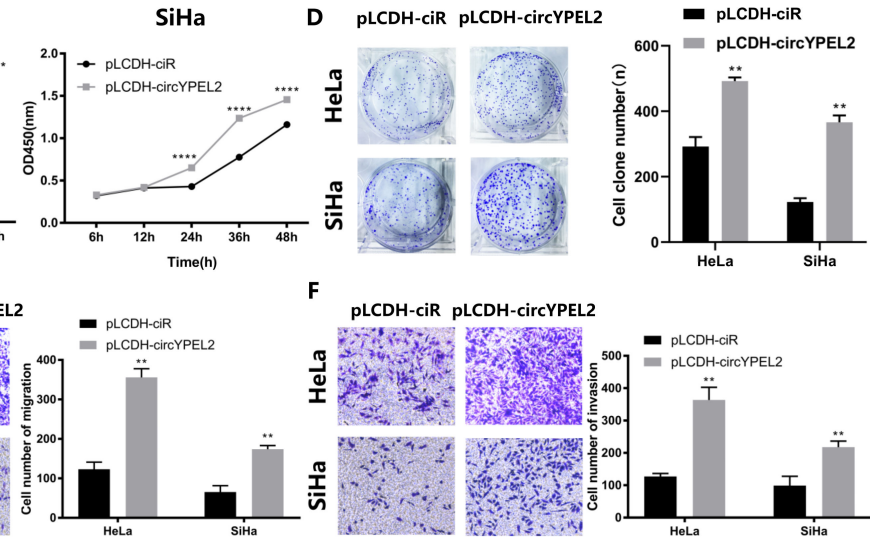

$\mathbf{F}$

PLCDH-ciR PLCDH-cirCYPEL2
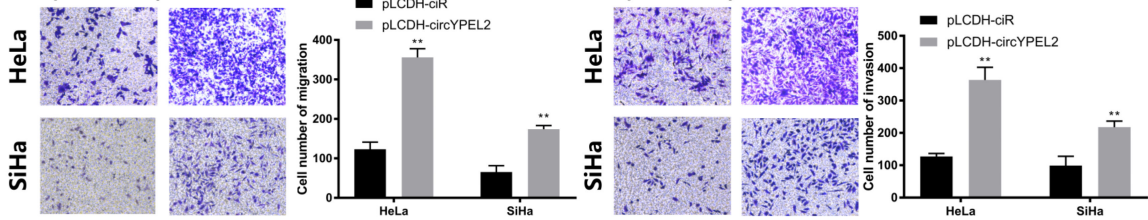

Figure 4. Overexpression of circYPEL2 promotes cell proliferation, migration and invasion. (A) Schematic illustration of the expression vector of circYPEL2. (B) Expression levels of circYPEL2 and YPEL2 mRNA in HeLa and SiHa cell lines transfected with the pLCDHircYPEL2 vector. The pLCDH-ciR vector was the negative control. (C) CCK-8 assays in HeLa and SiHa cell lines to detect cell viability at $0,6,12,24,36$ and $48 \mathrm{~h}$. (D) Clone formation assays in HeLa and SiHa cell lines to detect cell proliferation. (E,F) Transwell assay for cell migration and invasion ability in HeLa and SiHa cells. (Mean \pm SD of three experimental replicates are presented. ${ }^{* *}$ Represents $p<0.01$, $* * * *$ represents $p<0.0001)$.

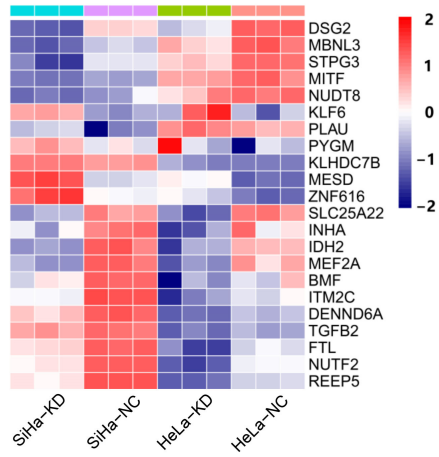

C

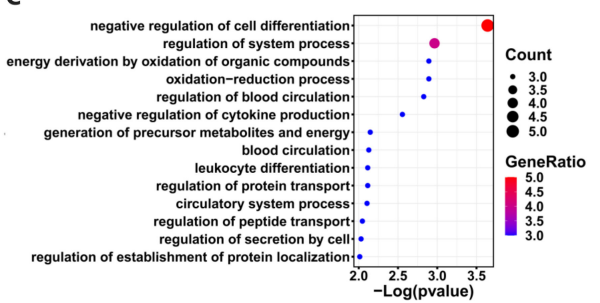

B

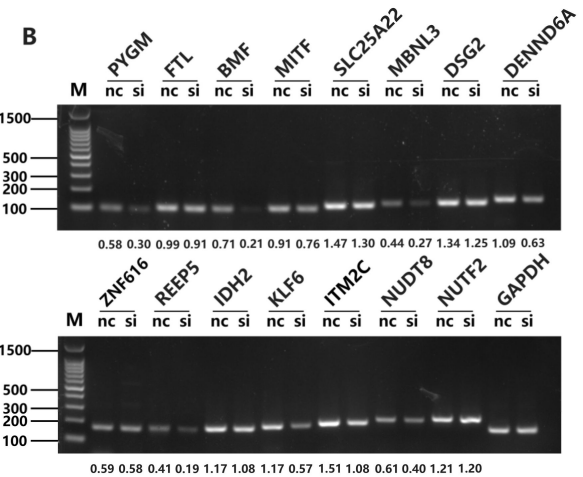

D

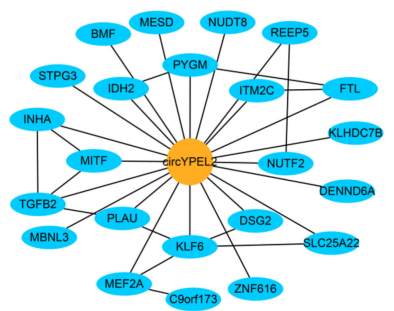

Figure 5. CircYPEL2 may regulate CC via downstream gene. (A) Clustering analysis of genes with significantly different expression in two cell lines treated with si-NC and si-circYPEL2 ( $\mid \log 2$ FoldChange $\mid>1$, adjusted $p$-value $<0.05$ ). TPM of genes were used for depicting the heatmap. (B) RT-PCR validation of differentially expressed genes. The nc represents si-NC and si represents si-circYPEL2. (C) Gene Ontology analysis of differentially expressed genes. (D) Regulatory network of circRNAs and coding genes. The blue and yellow nodes represent coding genes and circRNA, respectively. 


\section{Discussion}

As a new type of endogenous noncoding RNA, circRNAs are produced by precursor mRNA (premRNA) and have special structural stability [34]. The dysregulation of circRNAs in cancers has been widely reported, and accumulating evidence suggests that circRNAs are associated with tumor progression $[30,35]$ and circRNAs are potential biomarkers for tumor diagnosis, treatment and prognosis [36,37]. In this study, high throughput sequencing revealed a total of 59 differentially expressed circRNAs between CC and normal controls. We analyzed expression of these circRNAs and found that highly expressed circRNAs were concomitant with low expression of host genes, such as circYPEL2 and circFCHO2, indicating an independent expression pattern of circRNA, which was also revealed in previous work [38]. In addition, circRNAs were more stable than their host genes in CC cells (Figure 2C). These results suggest that circRNAs are potential robust biomarker than linear genes.

Gain and loss of function experiments suggested that circYPEL2 may act as an oncogene in CC by regulating cell proliferation, invasion and migration. We further identified the downstream genes of circYPEL2 by performing high-throughput sequencing on CC cell lines with knockdown of circYPEL2. Genes such as PYGM, BMF, MBNL3, DENND6A, REEP5, KLF6 and ITM2C are potentially regulated by circYPEL2 and involved in cancerspecific pathways [39-41], which are needed to be validated in further studies.

\section{Conclusions}

In summary, combining with high throughput sequencing and experiment analysis, we explored the CC-associated circRNAs and investigated the potential function of circYPEL2 in CC cells, which provided a novel view for understanding the development of CC and diagnosis.

Supplementary Materials: The following are available online at https:/ /www.mdpi.com/article/10 .3390/genes13010038/s1, Figure S1: CircYPEL2 is upregulated in cervical cancer, Table S1: Primers used in qRT-PCR \& RT-PCR analysis, Table S2: Differentially expressed genes enriched in GSEA hallmark pathways.

Author Contributions: Study concept and research design: Y.F., C.H. and H.L.; sample collection and processing: Y.F. and H.L.; sequencing and experiments: X.Z., S.Y., W.C., X.D., R.Z., H.Y., Y.F. and X.M.; writing of the manuscript: C.H., Y.F., X.Z. and S.Y.; funding recruitment: C.H. and H.L. All authors have read and agreed to the published version of the manuscript.

Funding: National Natural Science Foundation of China [81870129 and 82170170]; National Key R\&D Program of China [2018YFA0902400].

Institutional Review Board Statement: The study was approved by the Ethics Committee (Institutional Review Board) of Wuhan University Zhongnan Hospital, with ethical approval number (JC2019-023). All patients provided written informed consent for participation according to the Declaration of Helsinki and written informed consent for publication. Clinical information was retrieved from the medical records. All in vivo operations were carried out following the Guidelines for Animal Experimentation of Wuhan University. Our protocol was approved by the Ethics Committee for Animal Experimentation and was performed based on the Guidelines for Animal Experimentation of Wuhan University and the National Institute of Health Guide for the Care and Use of Laboratory Animals.

Informed Consent Statement: Informed consent was obtained from all subjects involved in the study. Written informed consent has been obtained from the patient(s) to publish this paper.

Data Availability Statement: All raw data were deposited in the Gene Expression Omnibus (GEO) repository (https:/ / www.ncbi.nlm.nih.gov / geo/query /acc.cgi, accession number GSE173112).

Acknowledgments: The author would also like to thank Wuhan University for financial support of this research.

Conflicts of Interest: The authors declare no conflict of interest. 


\section{References}

1. Bray, F.; Ferlay, J.; Soerjomataram, I.; Siegel, R.L.; Torre, L.A.; Jemal, A. Global cancer statistics 2018: GLOBOCAN estimates of incidence and mortality worldwide for 36 cancers in 185 countries. CA-A Cancer J. Clin. 2018, 68, 394-424. [CrossRef]

2. Munoz, N.; Bosch, F.X.; De Sanjosé, S.; Herrero, R.; Castellsagué, X.; Shah, K.V.; Snijders, P.J.; Meijer, C.J. Epidemiologic classification of human papillomavirus types associated with cervical cancer. N. Engl. J. Med. 2003, 348, 518-527. [CrossRef] [PubMed]

3. Ronco, G.; Dillner, J.; Elfström, K.M.; Tunesi, S.; Snijders, P.J.F.; Arbyn, M.; Kitchener, H.; Segnan, N.; Gilham, C.; Rossi, P.G.; et al. Efficacy of HPV-based screening for prevention of invasive cervical cancer: Follow-up of four European randomised controlled trials. Lancet 2014, 383, 524-532. [CrossRef]

4. Tsikouras, P.; Zervoudis, S.; Manav, B.; Tomara, E.; Iatrakis, G.; Romanidis, C.; Bothou, A.; Galazios, G. Cervical cancer: Screening, diagnosis and staging. J. Buon 2016, 21, 320-325.

5. Jeck, W.R.; Sorrentino, J.A.; Wang, K.; Slevin, M.K.; Burd, C.E.; Liu, J.; Marzluff, W.F.; Sharpless, N.E. Circular RNAs are abundant, conserved, and associated with ALU repeats. RNA 2013, 19, 141-157. [CrossRef]

6. Rybak-Wolf, A.; Stottmeister, C.; Glažar, P.; Jens, M.; Pino, N.; Giusti, S.; Hanan, M.; Behm, M.; Bartok, O.; Ashwal-Fluss, R.; et al Circular RNAs in the Mammalian Brain Are Highly Abundant, Conserved, and Dynamically Expressed. Mol. Cell 2015, 58, 870-885. [CrossRef]

7. Salzman, J.; Chen, R.E.; Olsen, M.N.; Wang, P.L.; Brown, P.O. Cell-Type Specific Features of Circular RNA Expression. PLoS Genet. 2013, 9, e1003777. [CrossRef]

8. Vo, J.N.; Cieslik, M.; Zhang, Y.; Shukla, S.; Xiao, L.; Zhang, Y.; Wu, Y.-M.; Dhanasekaran, S.M.; Engelke, C.G.; Cao, X.; et al. The Landscape of Circular RNA in Cancer. Cell 2019, 176, 869-881. [CrossRef] [PubMed]

9. Sun, Y.M.; Wang, W.-T.; Zeng, Z.-C.; Chen, T.-Q.; Han, C.; Pan, Q.; Huang, W.; Fang, K.; Sun, L.-Y.; Zhou, Y.-F.; et al. circMYBL2, a circRNA from MYBL2, regulates FLT3 translation by recruiting PTBP1 to promote FLT3-ITD AML progression. Blood 2019, 134, 1533-1546. [CrossRef] [PubMed]

10. Xu, X.; Zhang, J.; Tian, Y.; Gao, Y.; Dong, X.; Chen, W.; Yuan, X.; Yin, W.; Xu, J.; Chen, K.; et al. CircRNA inhibits DNA damage repair by interacting with host gene. Mol. Cancer 2020, 19, 128. [CrossRef]

11. Legnini, I.; Di Timoteo, G.; Rossi, F.; Morlando, M.; Briganti, F.; Sthandier, O.; Fatica, A.; Santini, T.; Andronache, A.; Wade, M.; et al. Circ-ZNF609 Is a Circular RNA that Can Be Translated and Functions in Myogenesis. Mol. Cell 2017, 66, 22-37.e9. [CrossRef]

12. Du, W.W.; Fang, L.; Yang, W.; Wu, N.; Awan, F.M.; Yang, Z.; Yang, B.B. Induction of tumor apoptosis through a circular RNA enhancing Foxo3 activity. Cell Death Differ. 2017, 24, 357-370. [CrossRef] [PubMed]

13. Han, D.; Li, J.; Wang, H.; Su, X.; Hou, J.; Gu, Y.; Qian, C.; Lin, Y.; Liu, X.; Huang, M.; et al. Circular RNA circMTO1 acts as the sponge of microRNA-9 to suppress hepatocellular carcinoma progression. Hepatology 2017, 66, 1151-1164. [CrossRef] [PubMed]

14. Li, J.F.; Huang, C.; Zou, Y.; Ye, J.; Yu, J.; Gui, Y. CircTLK1 promotes the proliferation and metastasis of renal cell carcinoma by sponging miR-136-5p. Mol. Cancer 2020, 19, 1-17. [CrossRef] [PubMed]

15. Lu, Q.; Liu, T.; Yang, R.; Zhao, X.; Guo, H. Circular RNA Circslc8a1 Acts as a Sponge of Mir-130b/Mir-494 in Suppressing Bladder Cancer Progression Via Regulating Pten. J. Urol. 2020, 203, E3. [CrossRef]

16. Ma, X.K.; Wang, M.-R.; Liu, C.-X.; Dong, R.; Carmichael, G.G.; Chen, L.-L.; Yang, L. CIRCexplorer3: A CLEAR Pipeline for Direct Comparison of Circular and Linear RNA Expression. Genom. Proteom. Bioinform. 2019, 17, 511-521. [CrossRef]

17. Dobin, A.; Davis, C.A.; Schlesinger, F.; Drenkow, J.; Zaleski, C.; Jha, S.; Batut, P.; Chaisson, M.; Gingeras, T.R. STAR: Ultrafast universal RNA-seq aligner. Bioinformatics 2013, 29, 15-21. [CrossRef]

18. Liao, Y.; Smyth, G.K.; Shi, W. featureCounts: An efficient general purpose program for assigning sequence reads to genomic features. Bioinformatics 2014, 30, 923-930. [CrossRef]

19. Wagner, G.P.; Kin, K.; Lynch, V.J. Measurement of mRNA abundance using RNA-seq data: RPKM measure is inconsistent among samples. Theory Biosci. 2012, 131, 281-285. [CrossRef]

20. Li, B.; Dewey, C.N. RSEM: Accurate transcript quantification from RNA-Seq data with or without a reference genome. BMC Bioinform. 2011, 12, 323. [CrossRef]

21. Love, M.I.; Huber, W.; Anders, S. Moderated estimation of fold change and dispersion for RNA-seq data with DESeq2. Genome Biol. 2014, 15, 1-21. [CrossRef]

22. Yu, G.C.; Wang, L.-G.; Han, Y.; He, Q.-Y. clusterProfiler: An R Package for Comparing Biological Themes Among Gene Clusters Omics-a J. Integr. Biol. 2012, 16, 284-287. [CrossRef] [PubMed]

23. Roomi, M.W.; Monterrey, J.C.; Kalinovsky, T.; Rath, M.; Niedzwiecki, A. In vitro modulation of MMP-2 and MMP-9 in human cervical and ovarian cancer cell lines by cytokines, inducers and inhibitors. Oncol. Rep. 2010, 23, 605-614.

24. Chen, H.F.; Gu, B.; Zhao, X.; Zhao, Y.; Huo, S.; Liu, X.; Lu, H. Circular RNA hsa_circ_0007364 increases cervical cancer progression through activating methionine adenosyltransferase II $\alpha$ (MAT2A) expression by restraining microRNA-101-5p. Bioengineered 2020, 11, 1269-1279. [CrossRef] [PubMed]

25. Li, M.; Xie, X.; Zhou, J.; Sheng, M.; Yin, X.; Ko, E.-A.; Zhou, T.; Gu, W. Quantifying circular RNA expression from RNA-seq data using model-based framework. Bioinformatics 2017, 33, 2131-2139. [CrossRef]

26. Yang, X.; Li, S.; Wu, Y.; Ge, F.; Chen, Y.; Xiong, Q. The circular RNA CDR1as regulate cell proliferation via TMED2 and TMED10. BMC Cancer 2020, 20, 312. [CrossRef] [PubMed] 
27. Vanaja, G.R.; Ramulu, H.G.; Kalle, A.M. Overexpressed HDAC8 in cervical cancer cells shows functional redundancy of tubulin deacetylation with HDAC6. Cell Commun. Signal 2018, 16, 20. [CrossRef]

28. Jinawath, N.; Shiao, M.-S.; Chanpanitkitchote, P.; Svasti, J.; Furukawa, Y.; Nakamura, Y. Enhancement of Migration and Invasion of Gastric Cancer Cells by IQGAP3. Biomolecules 2020, 10, 1194. [CrossRef]

29. Zhu, X.; Li, S. TET2 inhibits tumorigenesis of breast cancer cells by regulating caspase-4. Sci. Rep. 2018, 8, 16167. [CrossRef]

30. Xia, S.; Feng, J.; Chen, K.; Ma, Y.; Gong, J.; Cai, F.; Jin, Y.; Gao, Y.; Xia, L.; Chang, H.; et al. CSCD: A database for cancer-specific circular RNAs. Nucleic Acids Res. 2018, 46, D925-D929. [CrossRef] [PubMed]

31. Ashton, T.M.; McKenna, W.G.; Kunz-Schughart, L.A.; Higgins, G.S. Oxidative Phosphorylation as an Emerging Target in Cancer Therapy. Clin. Cancer Res. 2018, 24, 2482-2490. [CrossRef]

32. Jing, X.; Yang, F.; Shao, C.; Wei, K.; Xie, M.; Shen, H.; Shu, Y. Role of hypoxia in cancer therapy by regulating the tumor microenvironment. Mol. Cancer 2019, 18, 157. [CrossRef]

33. Rodic, S.; Vincent, M.D. Reactive oxygen species (ROS) are a key determinant of cancer's metabolic phenotype. Int. J. Cancer 2018, $142,440-448$.

34. Cocquerelle, C.; Mascrez, B.; Hétuin, D.; Bailleul, B. Mis-splicing yields circular RNA molecules. FASEB J. 1993, 7, 155-160. [CrossRef]

35. Qu, S.; Yang, X.; Li, X.; Wang, J.; Gao, Y.; Shang, R.; Sun, W.; Dou, K.; Li, H. Circular RNA: A new star of noncoding RNAs. Cancer Lett. 2015, 365, 141-148.

36. Kristensen, L.S.; Andersen, M.S.; Stagsted, L.V.W.; Ebbesen, K.K.; Hansen, T.B.; Kjems, J. The biogenesis, biology and characterization of circular RNAs. Nat. Rev. Genet. 2019, 20, 675-691. [CrossRef]

37. Wu, J.; Qi, X.; Liu, L.; Hu, X.; Liu, J.; Yang, J.; Yang, J.; Lu, L.; Zhang, Z.; Ma, S.; et al. Emerging Epigenetic Regulation of Circular RNAs in Human Cancer. Mol. Ther. Nucleic Acids 2019, 16, 589-596. [CrossRef]

38. Sui, W.G.; Gan, Q.; Chang, Y.; Ou, M.; Chen, J.; Lin, H.; Xue, W.; Wu, Y.; He, H.; Tang, D.; et al. Differential expression profile study and gene function analysis of maternal foetal-derived circRNA for screening for Down's syndrome. Exp. Ther. Med. 2020, 19, 1006-1016. [CrossRef]

39. Xie, X.; Huang, Y.; Chen, L.; Wang, J. miR-221 regulates proliferation and apoptosis of ovarian cancer cells by targeting BMF. Oncol. Lett. 2018, 16, 6697-6704.

40. Zhao, Y.; Qin, X.-P.; Lang, Y.-P.; Kou, D.; Shao, Z.-W. Circular RNA circ-SMAD7 promoted ovarian cancer cell proliferation and metastasis by suppressing KLF6. Eur. Rev. Med. Pharmacol. Sci. 2020, 24, 7563. [PubMed]

41. Han, X.T.; Jiang, J.-Q.; Li, M.-Z.; Cong, Q.-M. Circular RNA circ-ABCB10 promotes the proliferation and invasion of thyroid cancer by targeting KLF6. Eur Rev. Med. Pharmacol. Sci. 2020, 24, 1271-1277. 